\title{
Intra- and Inter-Molecular Singlet Fission in Covalently Linked Dimers
}

\author{
Xintian Feng ${ }^{a}$, David Casanova ${ }^{b, c}$, and Anna I. Krylov ${ }^{a}$ \\ ${ }^{a}$ Department of Chemistry, University of Southern California, Los Angeles, California 90089-0482 \\ ${ }^{b}$ Kimika Fakultatea, Euskal Herriko Unibertsitatea (UPV/EHU) \\ and Donostia International Physics Center (DIPC), P.K. 1072, 20018 Donostia, Euskadi, Spain \\ ${ }^{c}$ IKERBASQUE, Basque Foundation for Science, 48013 Bilbao, Euskadi, Spain
}

August 6, 2016

\section{Raw RAS-SF energies and energy correction}

Table S1: Lowest states in BET-B and (BET-B) $)_{2}$ computed with RAS-2SF and RAS-4SF, respectively. ME and EX excitation energies were corrected following Eqns. (3)-(9) from main text. $\mathrm{CR}_{1}$ and $\mathrm{CR}_{2}$ states are two lowest $\mathrm{CR}$ diabats of $\mathrm{BET}-\mathrm{B}$ computed using modified RAS-SF wave function.

\begin{tabular}{llllll}
\hline \hline System & State & Char. & Raw $\mathrm{E}_{e x}, \mathrm{eV}$ & Corrected $\mathrm{E}_{e x}, \mathrm{eV}$ & $f_{l}$ \\
\hline BET-B & $\mathrm{S}_{1}$ & $\mathrm{ME}$ & 3.208 & 2.493 & 0.000 \\
& $\mathrm{~S}_{2}$ & $\mathrm{EX}$ & 3.791 & 2.692 & 0.307 \\
& $\mathrm{~S}_{3}$ & $\mathrm{EX}$ & 4.124 & 3.087 & 0.932 \\
& $\mathrm{Q}_{1}$ & $\mathrm{ME}$ & 3.361 & 2.899 & \\
\hline & $\mathrm{CR}_{1}$ & $\mathrm{CR}$ & 4.306 & 2.613 & \\
& $\mathrm{CR}_{2}$ & $\mathrm{CR}$ & 4.411 & 2.718 & 0.000 \\
\hline \hline$(\text { BET-B })_{2}$ & $\mathrm{~S}_{1}$ & $\mathrm{ME}$ & 3.127 & 2.443 & 0.001 \\
& $\mathrm{~S}_{2}$ & $\mathrm{ME}$ & 3.127 & 2.441 & 0.000 \\
& $\mathrm{~S}_{3}$ & $\mathrm{ME}$ & 3.247 & 2.769 & 0.000 \\
& $\mathrm{~S}_{4}$ & $\mathrm{ME}$ & 3.266 & 2.777 & 0.000 \\
& $\mathrm{~S}_{5}$ & $\mathrm{ME}$ & 3.267 & 2.781 & 0.000 \\
& $\mathrm{~S}_{6}$ & $\mathrm{ME}$ & 3.286 & 2.790 & 0.000 \\
& $\mathrm{~S}_{7}$ & $\mathrm{EX}$ & 3.834 & 2.610 & 0.486 \\
& $\mathrm{~S}_{8}$ & $\mathrm{EX}$ & 3.922 & 2.643 & 0.000 \\
& $\mathrm{~S}_{9}$ & $\mathrm{EX}$ & 4.112 & 2.817 & 0.473 \\
& $\mathrm{~S}_{10}$ & $\mathrm{EX}$ & 4.127 & 2.728 & 0.170 \\
& $\mathrm{~S}_{11}$ & $\mathrm{EX}$ & 4.251 & 2.900 & 0.000 \\
\hline & $\mathrm{S}_{12}$ & $\mathrm{EX}$ & 4.388 & 2.897 & \\
& $\mathrm{~S}_{13}$ & $\mathrm{EX}$ & 4.398 & 2.895 & \\
\hline \hline
\end{tabular}




\subsection{Electronic states from $\omega$ B97X-D}

Table S2: Lowest states in BET-B and (BET-B) $)_{2}$ computed with $\omega$ B97X-D. CT states computed with C-DFT and $\mathrm{Q}_{1}$ corresponds to the UKS quintet.

\begin{tabular}{lll}
\hline \hline System & State & $\mathrm{E}_{e x}, \mathrm{eV}\left(f_{l}\right)$ \\
\hline BET-B & $\mathrm{S}_{1}$ & $2.692(0.168)$ \\
& $\mathrm{S}_{2}$ & $2.980(0.383)$ \\
& $\mathrm{Q}_{1}$ & 2.899 \\
& $\mathrm{CT}$ & 2.653 \\
\hline$(\text { BET-B })_{2}$ & $\mathrm{~S}_{1}$ & $2.612(0.000)$ \\
& $\mathrm{S}_{2}$ & $2.666(0.227)$ \\
& $\mathrm{S}_{3}$ & $2.854(0.011)$ \\
& $\mathrm{S}_{4}$ & $2.897(0.235)$ \\
& $\mathrm{S}_{5}$ & $3.025(0.000)$ \\
& $\mathrm{CT}$ & 3.704 \\
\hline \hline
\end{tabular}




\section{Calculations of model (BET-B $)_{2}$ structures}

At crystal (BET-B) $)_{2}$ geometry, the displacement along the long tetracene axis between the two middle tetracenes is $1.53 \AA$. When the two tetracenes are perfectly stacked, the shift is 0.00 $\AA$. The ground-state energy of this structure is $0.22 \mathrm{eV}$ higher than that of the original dimer. With the shift of $1.23 \AA$, the two middle tetracenes are offset by half of benzene ring (this structure is $0.02 \mathrm{eV}$ above the $\mathrm{X}$-ray dimer). The shift of $2.46 \AA$ corresponds to the structure in which the three benzenes of each middle tetracene are overlapped; this structure is $0.09 \mathrm{eV}$ higher than the original one.

Table S3: Corrected excitation energies $\left(\mathrm{E}_{e x}, \mathrm{eV}\right)$ and state characters of the ${ }^{1} \mathrm{ME}$ states.

\begin{tabular}{c|cc|cc|cc|cc} 
& \multicolumn{2}{|c|}{ zero-ring: $0.00 \AA$} & \multicolumn{2}{c|}{ half-ring: $1.23 \AA$} & \multicolumn{2}{c}{ X-ray: $1.53 \AA$} & \multicolumn{2}{c}{ one-ring: $2.46 \AA$} \\
State & $\mathrm{E}_{e x}$ & $\mathrm{TT} / \mathrm{TT} / \mathrm{CR}$ & $\mathrm{E}_{e x}$ & $\mathrm{TT} / \mathrm{TT} / \mathrm{CR}$ & $\mathrm{E}_{e x}$ & $\mathrm{TT} / \mathrm{TT} / \mathrm{CR}$ & $\mathrm{E}_{e x}$ & $\mathrm{TT} / \mathrm{TT} / \mathrm{CR}$ \\
\hline${ }^{1} \mathrm{ME}_{1}$ & 2.271 & $58 / 21 / 20$ & 2.445 & $80 / 00 / 18$ & 2.443 & $80 / 01 / 18$ & 2.355 & $75 / 05 / 19$ \\
${ }^{1} \mathrm{ME}_{2}$ & 2.267 & $59 / 20 / 20$ & 2.444 & $80 / 00 / 18$ & 2.441 & $80 / 01 / 18$ & 2.359 & $75 / 05 / 19$ \\
${ }^{1} \mathrm{ME}_{3}$ & 2.442 & $01 / 89 / 09$ & 2.772 & $00 / 98 / 01$ & 2.769 & $01 / 97 / 01$ & 2.597 & $00 / 95 / 04$ \\
${ }^{1} \mathrm{ME}_{4}$ & 2.664 & $19 / 74 / 06$ & 2.780 & $00 / 97 / 02$ & 2.777 & $01 / 96 / 02$ & 2.698 & $05 / 91 / 03$ \\
${ }^{1} \mathrm{ME}_{5}$ & 2.664 & $19 / 73 / 07$ & 2.786 & $00 / 97 / 02$ & 2.781 & $00 / 97 / 02$ & 2.697 & $05 / 91 / 03$ \\
${ }^{1} \mathrm{ME}_{6}$ & 2.779 & $00 / 97 / 02$ & 2.794 & $00 / 96 / 03$ & 2.790 & $00 / 96 / 03$ & 2.784 & $00 / 97 / 02$ \\
\hline
\end{tabular}

TT and TT' denote the \%weights of intra- and inter-molecular triplet-triplet configurations, respectively; CR denotes the \%weight of intra- and inter-molecular charge-resonance configurations.

Table S4: Corrected excitation energies $\left(\mathrm{E}_{e x}, \mathrm{eV}\right)$ and state characters of ${ }^{5} \mathrm{ME}$ states.

\begin{tabular}{c|cc|cc|cc|cc} 
& \multicolumn{2}{|c|}{ zero-ring: $0.00 \AA$} & \multicolumn{2}{|c|}{ half-ring: $1.23 \AA$} & \multicolumn{2}{c|}{ X-ray: $1.53 \AA$} & \multicolumn{2}{c}{ one-ring: $2.46 \AA$} \\
State & $\mathrm{E}_{e x}$ & $\mathrm{TT} / \mathrm{TT}$ & $\mathrm{E}_{\text {ex }}$ & $\mathrm{TT} / \mathrm{TT}$ & $\mathrm{E}_{e x}$ & $\mathrm{TT} / \mathrm{TT}$ & $\mathrm{E}_{e x}$ & $\mathrm{TT} / \mathrm{TT}$ \\
\hline${ }^{5} \mathrm{ME}_{1}$ & 2.624 & $42 / 42$ & 2.794 & $12 / 78$ & 2.788 & $22 / 68$ & 2.704 & $30 / 59$ \\
${ }^{5} \mathrm{ME}_{2}$ & 2.625 & $42 / 42$ & 2.806 & $47 / 42$ & 2.797 & $46 / 43$ & 2.713 & $44 / 44$ \\
${ }^{5} \mathrm{ME}_{3}$ & 2.772 & $07 / 84$ & 2.812 & $58 / 32$ & 2.809 & $41 / 49$ & 2.725 & $16 / 73$ \\
${ }^{5} \mathrm{ME}_{4}$ & 2.796 & $27 / 63$ & 2.823 & $43 / 47$ & 2.821 & $21 / 69$ & 2.769 & $43 / 48$ \\
${ }^{5} \mathrm{ME}_{5}$ & 2.799 & $45 / 45$ & 2.826 & $19 / 71$ & 2.823 & $44 / 47$ & 2.770 & $45 / 45$ \\
${ }^{5} \mathrm{ME}_{6}$ & 2.818 & $12 / 77$ & 2.854 & $01 / 89$ & 2.844 & $05 / 84$ & 2.817 & $01 / 89$ \\
\hline
\end{tabular}

TT and TT' denote the \%weights of intra- and inter-molecular triplet-triplet configurations, respectively. 
Table S5: Corrected excitation energies $\left(\mathrm{E}_{e x}, \mathrm{eV}\right)$ and state characters of singlet excitonic states.

\begin{tabular}{c|cc|cc|cc|cc} 
& \multicolumn{2}{|c|}{ zero-ring: $0.00 \AA$} & \multicolumn{2}{c|}{ half-ring: $1.23 \AA$} & \multicolumn{2}{c|}{ X-ray: $1.53 \AA$} & \multicolumn{2}{c}{ one-ring: $2.46 \AA$} \\
State & $\mathrm{E}_{e x}$ & LE/CR/CR & $\mathrm{E}_{e x}$ & LE/CR/CR' & $\mathrm{E}_{e x}$ & LE/CR/CR' & $\mathrm{E}_{e x}$ & LE/CR/CR' \\
\hline S7 & 1.873 & $42 / 02 / 44$ & 2.631 & $61 / 09 / 12$ & 2.610 & $60 / 09 / 14$ & 2.187 & $47 / 03 / 35$ \\
S8 & 2.660 & $51 / 28 / 05$ & 2.658 & $51 / 14 / 19$ & 2.643 & $51 / 13 / 21$ & 2.684 & $60 / 20 / 02$ \\
S9 & 2.736 & $45 / 31 / 07$ & 2.776 & $39 / 10 / 38$ & 2.817 & $45 / 10 / 29$ & 2.795 & $53 / 23 / 06$ \\
S10 & 2.556 & $01 / 03 / 94$ & 2.706 & $21 / 05 / 66$ & 2.728 & $24 / 06 / 60$ & 2.689 & $03 / 07 / 87$ \\
S11 & 2.828 & $22 / 46 / 18$ & 2.898 & $36 / 02 / 49$ & 2.900 & $32 / 01 / 56$ & 2.833 & $16 / 53 / 20$ \\
S12 & 2.867 & $27 / 43 / 14$ & 2.882 & $14 / 69 / 05$ & 2.897 & $15 / 72 / 01$ & 2.861 & $18 / 57 / 11$ \\
S13 & 3.181 & $55 / 17 / 05$ & 2.889 & $13 / 69 / 05$ & 2.895 & $11 / 75 / 01$ & 3.144 & $68 / 07 / 02$ \\
\hline
\end{tabular}

States are not ordered by excitation energy.

LE denotes the \%weight of local excitonic configurations; CR and CR' denote the \%weights of intra- and inter-molecular charge-resonance configurations. 
Table S6: Couplings $\|\gamma\|_{\text {sym }}^{2}$ between singlet ME states and two lowest excitonic states

\begin{tabular}{|c|c|c|c|c|}
\hline & zero-ring: $0.00 \AA$ & half-ring: $1.23 \AA$ & X-ray: $1.53 \AA$ & one-ring: $2.46 \AA$ \\
\hline $\mathrm{S} 7 \leftrightarrow{ }^{1} \mathrm{ME}_{1} /{ }^{1} \mathrm{ME}_{2}$ & $0.024 / 0.024$ & $0.025 / 0.025$ & $0.024 / 0.024$ & $0.025 / 0.025$ \\
\hline $\mathrm{S} 8 \leftrightarrow{ }^{1} \mathrm{ME}_{1} /{ }^{1} \mathrm{ME}_{2}$ & $0.049 / 0.055$ & $0.033 / 0.033$ & $0.030 / 0.031$ & $0.041 / 0.039$ \\
\hline $\mathrm{S} 7 \leftrightarrow{ }^{1} \mathrm{ME}_{3} /{ }^{1} \mathrm{ME}_{4}$ & $0.25 / 0.0073$ & $0.0053 / 0.0013$ & $0.0093 / 0.0014$ & $0.16 / 0.0049$ \\
\hline $\mathrm{S} 8 \leftrightarrow{ }^{1} \mathrm{ME}_{3} /{ }^{1} \mathrm{ME}_{4}$ & $0.0024 / 0.018$ & $0.0095 / 0.0032$ & $0.019 / 0.0029$ & $0.0021 / 0.0046$ \\
\hline $\mathrm{S} 7 \leftrightarrow{ }^{1} \mathrm{ME}_{5} /{ }^{1} \mathrm{ME}_{6}$ & $0.0070 / 0.00021$ & $0.018 / 0.024$ & $0.021 / 0.014$ & $0.0022 / 0.00014$ \\
\hline $\mathrm{S} 8 \leftrightarrow^{1} \mathrm{ME}_{5} /{ }^{1} \mathrm{ME}_{6}$ & $0.019 / 0.00060$ & $0.030 / 0.040$ & $0.042 / 0.027$ & $0.00012 / 0.00015$ \\
\hline${ }^{1} \mathrm{ME}_{3} \leftrightarrow{ }^{1} \mathrm{ME}_{1} /{ }^{1} \mathrm{ME}_{2}$ & $0.0056 / 0.0052$ & $0.0030 / 0.0039$ & $0.0027 / 0.0092$ & $0.0039 / 0.0016$ \\
\hline${ }^{1} \mathrm{ME}_{4} \leftrightarrow{ }^{1} \mathrm{ME}_{1} /{ }^{1} \mathrm{ME}_{2}$ & $0.020 / 0.25$ & $0.0073 / 0.0062$ & $0.016 / 0.0054$ & $0.075 / 0.012$ \\
\hline${ }^{1} \mathrm{ME}_{5} \leftrightarrow{ }^{1} \mathrm{ME}_{1} /{ }^{1} \mathrm{ME}_{2}$ & $0.25 / 0.022$ & $0.00084 / 0.0011$ & $0.00020 / 0.00032$ & $0.013 / 0.080$ \\
\hline${ }^{1} \mathrm{ME}_{6} \leftrightarrow{ }^{1} \mathrm{ME}_{1} /{ }^{1} \mathrm{ME}_{2}$ & $0.0020 / 0.0080$ & $0.0026 / 0.0025$ & $0.0029 / 0.0071$ & $0.0022 / 0.00080$ \\
\hline
\end{tabular}

States are not ordered by excitation energy.

The energies were corrected following Eqns. (4)-(7) from main text.

LE denotes the \%weight of local excitonic configurations; CR and CR' denote the \%weights of intra- and inter-molecular charge-resonance configurations, respectively.

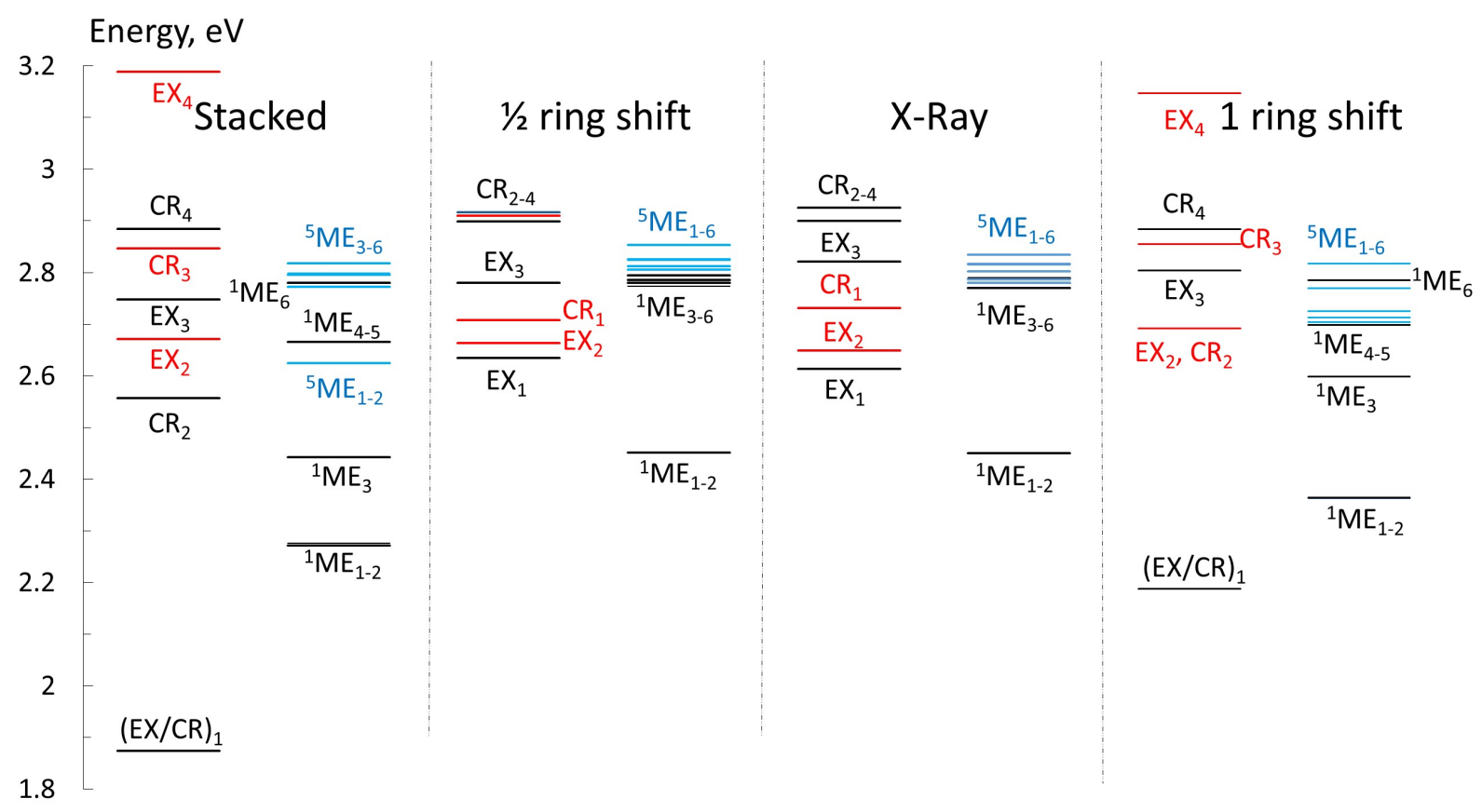

Figure S1: Excitation energies of (BET-B $)_{2}$ in model structures differing by the overlap between the middle tetracene moieties. Left: The tetracene rings are perfectly stacked (shift $0.00 \AA$ ). Middle: The tetracenes are shifted along the long axis by $1.23 \AA$ (half of benzene ring) and by $1.53 \AA$ (X-ray structure). Right: The tetracenes are offset by one benzene ring along the long axis (shift $2.46 \AA$ ). 
Table S7: Raw excitation energies $(\mathrm{eV})$ of all singlet states and six lowest quintet states State zero-ring: $0.00 \AA$ half-ring: $1.23 \AA$ X-ray: $1.53 \AA$ one-ring: $2.46 \AA$

\begin{tabular}{lllll}
\hline $\mathrm{S}_{0}^{a}$ & 0.221 & 0.013 & 0.000 & 0.090 \\
$\mathrm{~S}_{1}$ & 2.963 & 3.132 & 3.127 & 3.048 \\
$\mathrm{~S}_{2}$ & 2.964 & 3.132 & 3.127 & 3.048 \\
$\mathrm{~S}_{3}$ & 3.003 & 3.251 & 3.247 & 3.106 \\
$\mathrm{~S}_{4}$ & 3.172 & 3.269 & 3.266 & 3.199 \\
$\mathrm{~S}_{5}$ & 3.205 & 3.272 & 3.267 & 3.200 \\
$\mathrm{~S}_{6}$ & 3.207 & 3.291 & 3.286 & 3.270 \\
$\mathrm{~S}_{7}$ & 3.266 & 3.848 & 3.834 & 3.464 \\
$\mathrm{~S}_{8}$ & 3.957 & 3.936 & 3.922 & 3.929 \\
$\mathrm{~S}_{9}$ & 4.059 & 4.104 & 4.112 & 4.072 \\
$\mathrm{~S}_{10}$ & 4.068 & 4.122 & 4.127 & 4.189 \\
$\mathrm{~S}_{11}$ & 4.254 & 4.228 & 4.251 & 4.299 \\
$\mathrm{~S}_{12}$ & 4.255 & 4.369 & 4.388 & 4.308 \\
$\mathrm{~S}_{13}$ & 4.424 & 4.374 & 4.398 & 4.331 \\
\hline $\mathrm{Q}_{1}$ & 3.049 & 3.249 & 3.242 & 3.154 \\
$\mathrm{Q}_{2}$ & 3.049 & 3.258 & 3.249 & 3.159 \\
$\mathrm{Q}_{3}$ & 3.228 & 3.267 & 3.264 & 3.178 \\
$\mathrm{Q}_{4}$ & 3.252 & 3.278 & 3.278 & 3.226 \\
$\mathrm{Q}_{5}$ & 3.255 & 3.280 & 3.279 & 3.227 \\
$\mathrm{Q}_{6}$ & 3.270 & 3.307 & 3.297 & 3.270 \\
\hline \hline
\end{tabular}

$\overline{\bar{a}}$ : Relative to X-ray structure 


\section{Cartesian coordinates of relevant geometries}

\begin{tabular}{|c|c|c|c|}
\hline \multicolumn{4}{|c|}{$\begin{array}{l}\text { X-Ray BET-B dimer } \\
\text { Nuclear Repulsion }\end{array}$} \\
\hline C & 4.743687991 & -6.201925746 & -2.296318717 \\
\hline $\mathrm{H}$ & 4.570606309 & -7.221735433 & -2.643686471 \\
\hline $\mathrm{C}$ & 3.824845230 & -5.591702006 & -1.457066021 \\
\hline $\mathrm{H}$ & 2.919998404 & -6.115888600 & -1.146425534 \\
\hline $\mathrm{C}$ & 4.027625251 & -4.279421340 & -1.008527635 \\
\hline & 3.043285409 & -3.623011488 & -0.203512056 \\
\hline $\mathrm{C}$ & 2.206187482 & -3.042485199 & 0.436467782 \\
\hline C & 1.263520017 & -2.314573524 & 1.226934710 \\
\hline $\mathrm{C}$ & 0.390598748 & -3.003271246 & 2.095603744 \\
\hline C & 0.401852269 & -4.428478487 & 2.219821227 \\
\hline $\mathrm{H}$ & 1.104542255 & -5.000651840 & 1.612754141 \\
\hline C & -0.453096604 & -5.066569792 & 3.071533725 \\
\hline $\mathrm{H}$ & -0.432160654 & -6.155184881 & 3.147508263 \\
\hline C & -1.391167234 & -4.325625930 & 3.849415866 \\
\hline $\mathrm{H}$ & -2.072957043 & -4.859277517 & 4.513665326 \\
\hline $\mathrm{C}$ & -1.422000651 & -2.967772857 & 3.772584739 \\
\hline $\mathrm{H}$ & -2.126044031 & -2.390051370 & 4.375169142 \\
\hline $\mathrm{C}$ & -0.529986897 & -2.251813832 & 2.908711053 \\
\hline $\mathrm{C}$ & -0.527275071 & -0.860636131 & 2.852582387 \\
\hline $\mathrm{H}$ & -1.212286297 & -0.293906680 & 3.487306688 \\
\hline C & 0.360702759 & -0.154849747 & 2.017476656 \\
\hline $\mathrm{C}$ & 0.406496482 & 1.254943397 & 2.013444417 \\
\hline $\mathrm{H}$ & -0.264322060 & 1.811234089 & 2.671930965 \\
\hline $\mathrm{C}$ & 1.286720514 & 1.952523230 & 1.192675727 \\
\hline C & 1.372426826 & 3.384747436 & 1. 207212949 \\
\hline $\mathrm{H}$ & 0.709116888 & 3.942614111 & 1.871589963 \\
\hline $\mathrm{C}$ & 2.262595750 & 4.036980222 & 0.410006912 \\
\hline $\mathrm{H}$ & 2.314956968 & 5.127069762 & 0.427136521 \\
\hline $\mathrm{C}$ & 3.115443760 & 3.311315858 & -0.473680529 \\
\hline $\mathrm{H}$ & 3.809298408 & 3.857944187 & -1.114089775 \\
\hline $\mathrm{C}$ & 3.052632846 & 1.951587935 & -0.534308471 \\
\hline $\mathrm{H}$ & 3.704006673 & 1.390085736 & -1.206929868 \\
\hline $\mathrm{C}$ & 2.150213093 & 1.219165950 & 0.303680951 \\
\hline C & 2.112490298 & -0.172566213 & 0.302063742 \\
\hline $\mathrm{H}$ & 2.780201642 & -0.727338975 & -0.359346234 \\
\hline $\mathrm{C}$ & 1.253560758 & -0.888025920 & 1.156081126 \\
\hline C & 5.881928987 & -5.509031276 & -2.711754796 \\
\hline $\mathrm{H}$ & 6.596085931 & -5.988784783 & -3.382832099 \\
\hline $\mathrm{C}$ & 6.114926165 & -4.219215901 & -2.261112215 \\
\hline $\mathrm{H}$ & 7.016260052 & -3.682895997 & -2.561077004 \\
\hline $\mathrm{C}$ & 5.211806344 & -3.591306168 & -1.392497076 \\
\hline $\mathrm{C}$ & 5.489165325 & -2.289121666 & -0.867865910 \\
\hline
\end{tabular}




\begin{tabular}{|c|c|c|c|}
\hline C & 5.728657827 & -1.214065837 & -0.383907016 \\
\hline C & 5.943208167 & 0.075168527 & 0.195021543 \\
\hline $\mathrm{C}$ & 6.718076113 & 1.035509702 & -0.489233515 \\
\hline $\mathrm{C}$ & 7.317033836 & 0.770212171 & -1.761097962 \\
\hline $\mathrm{H}$ & 7.170478538 & -0.213094220 & -2.209850701 \\
\hline $\mathrm{C}$ & 8.059134707 & 1.720933058 & -2.400573028 \\
\hline $\mathrm{H}$ & 8.507673397 & 1.499125501 & -3.370518016 \\
\hline C & 8.267804510 & 3.000691555 & -1.806645373 \\
\hline $\mathrm{H}$ & 8.874921549 & 3.740157305 & -2.331127528 \\
\hline $\mathrm{C}$ & 7.701183918 & 3.298001576 & -0.606158843 \\
\hline $\mathrm{H}$ & 7.842101296 & 4.281409943 & -0.152826604 \\
\hline $\mathrm{C}$ & 6.896504920 & 2.340093847 & 0.093461124 \\
\hline C & 6.280336249 & 2.648412401 & 1.303340875 \\
\hline $\mathrm{H}$ & 6.394634366 & 3.650003223 & 1.723780479 \\
\hline $\mathrm{C}$ & 5.474785813 & 1.714269557 & 1.983420780 \\
\hline $\mathrm{C}$ & 4.798099414 & 2.052279107 & 3.173997885 \\
\hline $\mathrm{H}$ & 4.888979658 & 3.067754978 & 3.565837171 \\
\hline C & 3.998340881 & 1.131780424 & 3.843430674 \\
\hline $\mathrm{C}$ & 3.272380199 & 1.473288808 & 5.033043522 \\
\hline $\mathrm{H}$ & 3.353709057 & 2.490028141 & 5.423310751 \\
\hline $\mathrm{C}$ & 2.477622544 & 0.556732466 & 5.650806038 \\
\hline $\mathrm{H}$ & 1.922649604 & 0.831704799 & 6.550059618 \\
\hline C & 2.365875780 & -0.770717445 & 5.140678390 \\
\hline $\mathrm{H}$ & 1.725892416 & -1.488760669 & 5.655835593 \\
\hline $\mathrm{C}$ & 3.053993245 & -1.143317097 & 4.025266051 \\
\hline $\mathrm{H}$ & 2.972996693 & -2.158166991 & 3.630947324 \\
\hline $\mathrm{C}$ & 3.882817003 & -0.207004807 & 3.325692916 \\
\hline $\mathrm{C}$ & 4.546301178 & -0.548856431 & 2.150418890 \\
\hline $\mathrm{H}$ & 4.441366971 & -1.559109664 & 1.751005184 \\
\hline $\mathrm{C}$ & 5.326122341 & 0.385758584 & 1.445180560 \\
\hline $\mathrm{C}$ & -4.743687991 & 6.201925746 & 2.296318717 \\
\hline $\mathrm{H}$ & -4.570606309 & 7.221735433 & 2.643686471 \\
\hline C & -3.824845230 & 5.591702006 & 1.457066021 \\
\hline $\mathrm{H}$ & -2.919998404 & 6.115888600 & 1.146425534 \\
\hline $\mathrm{C}$ & -4.027625251 & 4.279421340 & 1.008527635 \\
\hline $\mathrm{C}$ & -3.043285409 & 3.623011488 & 0.203512056 \\
\hline $\mathrm{C}$ & -2.206187482 & 3.042485199 & -0.436467782 \\
\hline $\mathrm{C}$ & -1.263520017 & 2.314573524 & -1.226934710 \\
\hline $\mathrm{C}$ & -0.390598748 & 3.003271246 & -2.095603744 \\
\hline $\mathrm{C}$ & -0.401852269 & 4.428478487 & -2.219821227 \\
\hline $\mathrm{H}$ & -1.104542255 & 5.000651840 & -1.612754141 \\
\hline $\mathrm{C}$ & 0.453096604 & 5.066569792 & -3.071533725 \\
\hline $\mathrm{H}$ & 0.432160654 & 6.155184881 & -3.147508263 \\
\hline $\mathrm{C}$ & 1.391167234 & 4.325625930 & -3.849415866 \\
\hline $\mathrm{H}$ & 2.072957043 & 4.859277517 & -4.513665326 \\
\hline $\mathrm{C}$ & 1.422000651 & 2.967772857 & -3.772584739 \\
\hline
\end{tabular}




\begin{tabular}{|c|c|c|c|}
\hline $\mathrm{H}$ & 2.126044031 & 2.390051370 & -4.375169142 \\
\hline C & 0.529986897 & 2.251813832 & -2.908711053 \\
\hline $\mathrm{C}$ & 0.527275071 & 0.860636131 & -2.852582387 \\
\hline $\mathrm{H}$ & 1.212286297 & 0.293906680 & -3.487306688 \\
\hline$C$ & -0.360702759 & 0.154849747 & -2.017476656 \\
\hline $\mathrm{C}$ & -0.406496482 & -1.254943397 & -2.013444417 \\
\hline $\mathrm{H}$ & 0.264322060 & -1.811234089 & -2.671930965 \\
\hline C & -1.286720514 & -1.952523230 & -1.192675727 \\
\hline $\mathrm{C}$ & -1.372426826 & -3.384747436 & -1.207212949 \\
\hline $\mathrm{H}$ & -0.709116888 & -3.942614111 & -1.871589963 \\
\hline $\mathrm{C}$ & -2.262595750 & -4.036980222 & -0.410006912 \\
\hline $\mathrm{H}$ & -2.314956968 & -5.127069762 & -0.427136521 \\
\hline C & -3.115443760 & -3.311315858 & 0.473680529 \\
\hline $\mathrm{H}$ & -3.809298408 & -3.857944187 & 1.114089775 \\
\hline $\mathrm{C}$ & -3.052632846 & -1.951587935 & 0.534308471 \\
\hline $\mathrm{H}$ & -3.704006673 & -1.390085736 & 1.206929868 \\
\hline $\mathrm{C}$ & -2.150213093 & -1.219165950 & -0.303680951 \\
\hline C & -2.112490298 & 0.172566213 & -0.302063742 \\
\hline $\mathrm{H}$ & -2.780201642 & 0.727338975 & 0.359346234 \\
\hline $\mathrm{C}$ & -1.253560758 & 0.888025920 & -1.156081126 \\
\hline $\mathrm{C}$ & -5.881928987 & 5.509031276 & 2.711754796 \\
\hline $\mathrm{H}$ & -6.596085931 & 5.988784783 & 3.382832099 \\
\hline C & -6.114926165 & 4.219215901 & 2.261112215 \\
\hline $\mathrm{H}$ & -7.016260052 & 3.682895997 & 2.561077004 \\
\hline $\mathrm{C}$ & -5.211806344 & 3.591306168 & 1.392497076 \\
\hline $\mathrm{C}$ & -5.489165325 & 2.289121666 & 0.867865910 \\
\hline $\mathrm{C}$ & -5.728657827 & 1.214065837 & 0.383907016 \\
\hline C & -5.943208167 & -0.075168527 & -0.195021543 \\
\hline $\mathrm{C}$ & -6.718076113 & -1.035509702 & 0.489233515 \\
\hline $\mathrm{C}$ & -7.317033836 & -0.770212171 & 1.761097962 \\
\hline $\mathrm{H}$ & -7.170478538 & 0.213094220 & 2.209850701 \\
\hline $\mathrm{C}$ & -8.059134707 & -1.720933058 & 2.400573028 \\
\hline $\mathrm{H}$ & -8.507673397 & -1.499125501 & 3.370518016 \\
\hline $\mathrm{C}$ & -8.267804510 & -3.000691555 & 1.806645373 \\
\hline $\mathrm{H}$ & -8.874921549 & -3.740157305 & 2.331127528 \\
\hline $\mathrm{C}$ & -7.701183918 & -3.298001576 & 0.606158843 \\
\hline $\mathrm{H}$ & -7.842101296 & -4.281409943 & 0.152826604 \\
\hline $\mathrm{C}$ & -6.896504920 & -2.340093847 & -0.093461124 \\
\hline $\mathrm{C}$ & -6.280336249 & -2.648412401 & -1.303340875 \\
\hline $\mathrm{H}$ & -6.394634366 & -3.650003223 & -1.723780479 \\
\hline $\mathrm{C}$ & -5.474785813 & -1.714269557 & -1.983420780 \\
\hline $\mathrm{C}$ & -4.798099414 & -2.052279107 & -3.173997885 \\
\hline $\mathrm{H}$ & -4.888979658 & -3.067754978 & -3.565837171 \\
\hline $\mathrm{C}$ & -3.998340881 & -1.131780424 & -3.843430674 \\
\hline $\mathrm{C}$ & -3.272380199 & -1.473288808 & -5.033043522 \\
\hline $\mathrm{H}$ & -3.353709057 & -2.490028141 & -5.423310751 \\
\hline
\end{tabular}




$\begin{array}{llrl}\mathrm{C} & -2.477622544 & -0.556732466 & -5.650806038 \\ \mathrm{H} & -1.922649604 & -0.831704799 & -6.550059618 \\ \mathrm{C} & -2.365875780 & 0.770717445 & -5.140678390 \\ \mathrm{H} & -1.725892416 & 1.488760669 & -5.655835593 \\ \mathrm{C} & -3.053993245 & 1.143317097 & -4.025266051 \\ \mathrm{H} & -2.972996693 & 2.158166991 & -3.630947324 \\ \mathrm{C} & -3.882817003 & 0.207004807 & -3.325692916 \\ \mathrm{C} & -4.546301178 & 0.548856431 & -2.150418890 \\ \mathrm{H} & -4.441366971 & 1.559109664 & -1.751005184 \\ \mathrm{C} & -5.326122341 & -0.385758584 & -1.445180560\end{array}$

Perfect-stacked BET-B dimer

Nuclear Repulsion Energy $=16101.8252305983$ hartrees

$\begin{array}{lrrr}\mathrm{C} & 4.093935687 & -6.753219401 & -0.368140144 \\ \mathrm{H} & 3.754454868 & -7.789396184 & -0.406322358 \\ \mathrm{C} & 3.261719360 & -5.784190832 & 0.169809424 \\ \mathrm{H} & 2.271498113 & -6.051313319 & 0.541875445 \\ \mathrm{C} & 3.668437231 & -4.444161369 & 0.227155561 \\ \mathrm{C} & 2.780908271 & -3.432263393 & 0.713163516 \\ \mathrm{C} & 2.031631357 & -2.564389398 & 1.077439154 \\ \mathrm{C} & 1.197886876 & -1.496019340 & 1.532159815 \\ \mathrm{C} & 0.188062509 & -1.746083578 & 2.485367352 \\ \mathrm{C} & -0.048813952 & -3.049696903 & 3.025047022 \\ \mathrm{H} & 0.573308820 & -3.875716841 & 2.678462697 \\ \mathrm{C} & -1.031664604 & -3.260083620 & 3.948764669 \\ \mathrm{H} & -1.196663275 & -4.263000394 & 4.346021595 \\ \mathrm{C} & -1.858183516 & -2.184001370 & 4.388069141 \\ \mathrm{H} & -2.647922331 & -2.374654566 & 5.116539995 \\ \mathrm{C} & -1.653870876 & -0.926271993 & 3.911632526 \\ \mathrm{H} & -2.278343688 & -0.095412737 & 4.248914698 \\ \mathrm{C} & -0.620775826 & -0.652002300 & 2.956617153 \\ \mathrm{C} & -0.378527829 & 0.638305570 & 2.492985094 \\ \mathrm{H} & -0.985996783 & 1.465733412 & 2.868725699 \\ \mathrm{C} & 0.647771120 & 0.912409877 & 1.567988919 \\ \mathrm{C} & 0.933648485 & 2.229615079 & 1.152206979 \\ \mathrm{H} & 0.340278772 & 3.051062251 & 1.561115065 \\ \mathrm{C} & 1.950371053 & 2.501556622 & 0.242593749 \\ \mathrm{C} & 2.278674177 & 3.839484429 & -0.158640998 \\ \mathrm{H} & 1.695812148 & 4.664697523 & 0.257199040 \\ \mathrm{C} & 3.296558778 & 4.074487853 & -1.031584361 \\ \mathrm{H} & 3.539437953 & 5.096346584 & -1.331361532 \\ \mathrm{C} & 4.045769463 & 2.993341688 & -1.583533914 \\ \mathrm{H} & 4.849689708 & 3.200429690 & -2.292285108 \\ \mathrm{C} & 3.754123403 & 1.706026242 & -1.245536130 \\ \mathrm{H} & 4.325229719 & 0.874760108 & -1.665339288 \\ \mathrm{C} & 2.708949646 & 1.409946902 & -0.311202951\end{array}$




\begin{tabular}{|c|c|c|c|}
\hline C & 2.434315006 & 0.106774502 & 0.094582150 \\
\hline $\mathrm{H}$ & 3.023367454 & -0.714057844 & -0.318433261 \\
\hline $\mathrm{C}$ & 1.434252018 & -0.175041008 & 1.042971456 \\
\hline $\mathrm{C}$ & 5.348601492 & -6.400897590 & -0.868105648 \\
\hline $\mathrm{H}$ & 5.999098933 & -7.161355069 & -1.303422878 \\
\hline $\mathrm{C}$ & 5.781157206 & -5.085916081 & -0.799481242 \\
\hline $\mathrm{H}$ & 6.769433783 & -4.804341319 & -1.165643693 \\
\hline $\mathrm{C}$ & 4.966332116 & -4.093669892 & -0.237435092 \\
\hline $\mathrm{C}$ & 5.441884807 & -2.751169652 & -0.098176027 \\
\hline $\mathrm{C}$ & 5.842921014 & -1.628891859 & 0.066081152 \\
\hline $\mathrm{C}$ & 6.252372097 & -0.272479590 & 0.255318922 \\
\hline $\mathrm{C}$ & 7.205058187 & 0.304123714 & -0.611167278 \\
\hline $\mathrm{C}$ & 7.797521103 & -0.424394776 & -1.690528574 \\
\hline $\mathrm{H}$ & 7.495378669 & -1.461984619 & -1.838719946 \\
\hline $\mathrm{C}$ & 8.714612199 & 0.161799528 & -2.514441450 \\
\hline $\mathrm{H}$ & 9.156018587 & -0.408129238 & -3.334204438 \\
\hline $\mathrm{C}$ & 9.116089666 & 1.514774609 & -2.308652159 \\
\hline $\mathrm{H}$ & 9.859178434 & 1.963879858 & -2.970211801 \\
\hline $\mathrm{C}$ & 8.563624575 & 2.246693639 & -1.303815402 \\
\hline $\mathrm{H}$ & 8.859532354 & 3.287577203 & -1.151582952 \\
\hline $\mathrm{C}$ & 7.581421830 & 1.681580839 & -0.426040432 \\
\hline $\mathrm{C}$ & 6.981696079 & 2.434213319 & 0.580143720 \\
\hline $\mathrm{H}$ & 7.257367489 & 3.486196928 & 0.691825891 \\
\hline $\mathrm{C}$ & 6.003429345 & 1.885605235 & 1.432239020 \\
\hline $\mathrm{C}$ & 5.349914337 & 2.669408583 & 2.405994240 \\
\hline $\mathrm{H}$ & 5.606361642 & 3.729065872 & 2.483605865 \\
\hline $\mathrm{C}$ & 4.380081150 & 2.129499129 & 3.244441117 \\
\hline C & 3.678684221 & 2.923750725 & 4.211928775 \\
\hline $\mathrm{H}$ & 3.926098734 & 3.985285497 & 4.286795829 \\
\hline $\mathrm{C}$ & 2.716398325 & 2.371007702 & 5.000488721 \\
\hline $\mathrm{H}$ & 2.176280147 & 2.989375419 & 5.719829557 \\
\hline $\mathrm{C}$ & 2.399047648 & 0.984134025 & 4.896995844 \\
\hline $\mathrm{H}$ & 1.625842553 & 0.557614604 & 5.538594930 \\
\hline $\mathrm{C}$ & 3.055076142 & 0.189043019 & 4.005964004 \\
\hline $\mathrm{H}$ & 2.807302505 & -0.871666547 & 3.922811316 \\
\hline $\mathrm{C}$ & 4.057363977 & 0.730215719 & 3.136721056 \\
\hline $\mathrm{C}$ & 4.696646577 & -0.050528047 & 2.177502755 \\
\hline $\mathrm{H}$ & 4.425750019 & -1.104401013 & 2.090560342 \\
\hline $\mathrm{C}$ & 5.650610531 & 0.494730914 & 1.299076427 \\
\hline $\mathrm{C}$ & -4.093935687 & 6.753219401 & 0.368140144 \\
\hline $\mathrm{H}$ & -3.754454868 & 7.789396184 & 0.406322358 \\
\hline $\mathrm{C}$ & -3.261719360 & 5.784190832 & -0.169809424 \\
\hline $\mathrm{H}$ & -2.271498113 & 6.051313319 & -0.541875445 \\
\hline $\mathrm{C}$ & -3.668437231 & 4.444161369 & -0.227155561 \\
\hline $\mathrm{C}$ & -2.780908271 & 3.432263393 & -0.713163516 \\
\hline $\mathrm{C}$ & -2.031631357 & 2.564389398 & -1.077439154 \\
\hline
\end{tabular}




\begin{tabular}{|c|c|c|c|}
\hline $\mathrm{C}$ & -1.197886876 & 1.496019340 & -1.532159815 \\
\hline $\mathrm{C}$ & -0.188062509 & 1.746083578 & -2.485367352 \\
\hline $\mathrm{C}$ & 0.048813952 & 3.049696903 & -3.025047022 \\
\hline $\mathrm{H}$ & -0.573308820 & 3.875716841 & -2.678462697 \\
\hline $\mathrm{C}$ & 1.031664604 & 3.260083620 & -3.948764669 \\
\hline $\mathrm{H}$ & 1.196663275 & 4.263000394 & -4.346021595 \\
\hline $\mathrm{C}$ & 1.858183516 & 2.184001370 & -4.388069141 \\
\hline $\mathrm{H}$ & 2.647922331 & 2.374654566 & -5.116539995 \\
\hline C & 1.653870876 & 0.926271993 & -3.911632526 \\
\hline $\mathrm{H}$ & 2.278343688 & 0.095412737 & -4.248914698 \\
\hline $\mathrm{C}$ & 0.620775826 & 0.652002300 & -2.956617153 \\
\hline $\mathrm{C}$ & 0.378527829 & -0.638305570 & -2.492985094 \\
\hline $\mathrm{H}$ & 0.985996783 & -1.465733412 & -2.868725699 \\
\hline $\mathrm{C}$ & -0.647771120 & -0.912409877 & -1.567988919 \\
\hline $\mathrm{C}$ & -0.933648485 & -2.229615079 & -1.152206979 \\
\hline $\mathrm{H}$ & -0.340278772 & -3.051062251 & -1.561115065 \\
\hline $\mathrm{C}$ & -1.950371053 & -2.501556622 & -0.242593749 \\
\hline $\mathrm{C}$ & -2.278674177 & -3.839484429 & 0.158640998 \\
\hline $\mathrm{H}$ & -1.695812148 & -4.664697523 & -0.257199040 \\
\hline $\mathrm{C}$ & -3.296558778 & -4.074487853 & 1.031584361 \\
\hline $\mathrm{H}$ & -3.539437953 & -5.096346584 & 1.331361532 \\
\hline $\mathrm{C}$ & -4.045769463 & -2.993341688 & 1.583533914 \\
\hline $\mathrm{H}$ & -4.849689708 & -3.200429690 & 2.292285108 \\
\hline $\mathrm{C}$ & -3.754123403 & -1.706026242 & 1.245536130 \\
\hline $\mathrm{H}$ & -4.325229719 & -0.874760108 & 1.665339288 \\
\hline $\mathrm{C}$ & -2.708949646 & -1.409946902 & 0.311202951 \\
\hline $\mathrm{C}$ & -2.434315006 & -0.106774502 & -0.094582150 \\
\hline $\mathrm{H}$ & -3.023367454 & 0.714057844 & 0.318433261 \\
\hline $\mathrm{C}$ & -1.434252018 & 0.175041008 & -1.042971456 \\
\hline $\mathrm{C}$ & -5.348601492 & 6.400897590 & 0.868105648 \\
\hline $\mathrm{H}$ & -5.999098933 & 7.161355069 & 1.303422878 \\
\hline $\mathrm{C}$ & -5.781157206 & 5.085916081 & 0.799481242 \\
\hline $\mathrm{H}$ & -6.769433783 & 4.804341319 & 1.165643693 \\
\hline $\mathrm{C}$ & -4.966332116 & 4.093669892 & 0.237435092 \\
\hline $\mathrm{C}$ & -5.441884807 & 2.751169652 & 0.098176027 \\
\hline $\mathrm{C}$ & -5.842921014 & 1.628891859 & -0.066081152 \\
\hline $\mathrm{C}$ & -6.252372097 & 0.272479590 & -0.255318922 \\
\hline $\mathrm{C}$ & -7.205058187 & -0.304123714 & 0.611167278 \\
\hline $\mathrm{C}$ & -7.797521103 & 0.424394776 & 1.690528574 \\
\hline $\mathrm{H}$ & -7.495378669 & 1.461984619 & 1.838719946 \\
\hline $\mathrm{C}$ & -8.714612199 & -0.161799528 & 2.514441450 \\
\hline $\mathrm{H}$ & -9.156018587 & 0.408129238 & 3.334204438 \\
\hline $\mathrm{C}$ & -9.116089666 & -1.514774609 & 2.308652159 \\
\hline $\mathrm{H}$ & -9.859178434 & -1.963879858 & 2.970211801 \\
\hline $\mathrm{C}$ & -8.563624575 & -2.246693639 & 1.303815402 \\
\hline $\mathrm{H}$ & -8.859532354 & -3.287577203 & 1. 151582952 \\
\hline
\end{tabular}




$\begin{array}{lrrr}\mathrm{C} & -7.581421830 & -1.681580839 & 0.426040432 \\ \mathrm{C} & -6.981696079 & -2.434213319 & -0.580143720 \\ \mathrm{H} & -7.257367489 & -3.486196928 & -0.691825891 \\ \mathrm{C} & -6.003429345 & -1.885605235 & -1.432239020 \\ \mathrm{C} & -5.349914337 & -2.669408583 & -2.405994240 \\ \mathrm{H} & -5.606361642 & -3.729065872 & -2.483605865 \\ \mathrm{C} & -4.380081150 & -2.129499129 & -3.244441117 \\ \mathrm{C} & -3.678684221 & -2.923750725 & -4.211928775 \\ \mathrm{H} & -3.926098734 & -3.985285497 & -4.286795829 \\ \mathrm{C} & -2.716398325 & -2.371007702 & -5.000488721 \\ \mathrm{H} & -2.176280147 & -2.989375419 & -5.719829557 \\ \mathrm{C} & -2.399047648 & -0.984134025 & -4.896995844 \\ \mathrm{H} & -1.625842553 & -0.557614604 & -5.538594930 \\ \mathrm{C} & -3.055076142 & -0.189043019 & -4.005964004 \\ \mathrm{H} & -2.807302505 & 0.871666547 & -3.922811316 \\ \mathrm{C} & -4.057363977 & -0.730215719 & -3.136721056 \\ \mathrm{C} & -4.696646577 & 0.050528047 & -2.177502755 \\ \mathrm{H} & -4.425750019 & 1.104401013 & -2.090560342 \\ \mathrm{C} & -5.650610531 & -0.494730914 & -1.299076427\end{array}$

Half-Ring shifted BET-B dimer

Nuclear Repulsion Energy $=16142.2918460569$ hartrees

$\begin{array}{rrrr}\mathrm{C} & 4.580984219 & -6.395866582 & -1.877163038 \\ \mathrm{H} & 4.368677595 & -7.429265402 & -2.156751498 \\ \mathrm{C} & 3.678702475 & -5.695644598 & -1.091945267 \\ \mathrm{H} & 2.751950469 & -6.162697548 & -0.756047514 \\ \mathrm{C} & 3.927893731 & -4.364569962 & -0.730654527 \\ \mathrm{C} & 2.962267385 & -3.618142680 & 0.016514111 \\ \mathrm{C} & 2.142357437 & -2.964161881 & 0.605899126 \\ \mathrm{C} & 1.221267909 & -2.149018076 & 1.334219095 \\ \mathrm{C} & 0.314405201 & -2.743525871 & 2.237016136 \\ \mathrm{C} & 0.269428598 & -4.156405635 & 2.457289107 \\ \mathrm{H} & 0.954779059 & -4.789841657 & 1.893686950 \\ \mathrm{C} & -0.617363807 & -4.702293598 & 3.339965622 \\ \mathrm{H} & -0.637531948 & -5.783517163 & 3.488322116 \\ \mathrm{C} & -1.533240375 & -3.875186117 & 4.054904965 \\ \mathrm{H} & -2.243612040 & -4.332175700 & 4.746531732 \\ \mathrm{C} & -1.510868336 & -2.525495644 & 3.886222402 \\ \mathrm{H} & -2.198130411 & -1.884842122 & 4.443297521 \\ \mathrm{C} & -0.583919006 & -1.904207649 & 2.986594826 \\ \mathrm{C} & -0.526931499 & -0.521159911 & 2.836713018 \\ \mathrm{H} & -1.196250025 & 0.108790502 & 3.427157024 \\ \mathrm{C} & 0.395326485 & 0.092093058 & 1.966424771 \\ \mathrm{C} & 0.495600433 & 1.495603775 & 1.867769643 \\ \mathrm{H} & -0.159343406 & 2.116229696 & 2.482636056 \\ \mathrm{C} & 1.409661294 & 2.101942826 & 1.012247048\end{array}$




\begin{tabular}{|c|c|c|c|}
\hline $\mathrm{C}$ & 1.550511750 & 3.527563880 & 0.931075403 \\
\hline $\mathrm{H}$ & 0.902210472 & 4.148837914 & 1.551589790 \\
\hline $\mathrm{C}$ & 2.472539825 & 4.089916286 & 0.102238999 \\
\hline $\mathrm{H}$ & 2.565664386 & 5.176365514 & 0.049968648 \\
\hline $\mathrm{C}$ & 3.304828003 & 3.274088920 & -0.720296160 \\
\hline $\mathrm{H}$ & 4.027682067 & 3.746399249 & -1.387745474 \\
\hline $\mathrm{C}$ & 3.190081747 & 1.916745925 & -0.689730267 \\
\hline $\mathrm{H}$ & 3.824897123 & 1.290361626 & -1.319090693 \\
\hline $\mathrm{C}$ & 2.252337338 & 1.277681673 & 0.185062254 \\
\hline $\mathrm{C}$ & 2.160878023 & -0.108512583 & 0.276957380 \\
\hline $\mathrm{H}$ & 2.810616317 & -0.729052068 & -0.341311157 \\
\hline $\mathrm{C}$ & 1.267097045 & -0.731227008 & 1.167109360 \\
\hline $\mathrm{C}$ & 5.748936404 & -5.776457867 & -2.324938638 \\
\hline $\mathrm{H}$ & 6.447818054 & -6.329454350 & -2.953978760 \\
\hline $\mathrm{C}$ & 6.027433403 & -4.468783672 & -1.959674704 \\
\hline $\mathrm{H}$ & 6.951227911 & -3.989545259 & -2.286323813 \\
\hline $\mathrm{C}$ & 5.141274642 & -3.749531560 & -1.146171568 \\
\hline $\mathrm{C}$ & 5.463893553 & -2.426183563 & -0.707403455 \\
\hline $\mathrm{C}$ & 5.740274234 & -1.330601954 & -0.294326509 \\
\hline $\mathrm{C}$ & 5.999132541 & -0.014095070 & 0.198742828 \\
\hline $\mathrm{C}$ & 6.816814585 & 0.867476282 & -0.539571818 \\
\hline $\mathrm{C}$ & 7.416785598 & 0.493888575 & -1.783452566 \\
\hline $\mathrm{H}$ & 7.234781308 & -0.510123721 & -2.169107978 \\
\hline $\mathrm{C}$ & 8.200941398 & 1.370152760 & -2.476830548 \\
\hline $\mathrm{H}$ & 8.647367537 & 1.063849999 & -3.424491542 \\
\hline $\mathrm{C}$ & 8.453419203 & 2.678454166 & -1.968227218 \\
\hline $\mathrm{H}$ & 9.092468344 & 3.357342533 & -2.535330128 \\
\hline $\mathrm{C}$ & 7.887655615 & 3.077883361 & -0.797335279 \\
\hline $\mathrm{H}$ & 8.062995499 & 4.083467189 & -0.407010734 \\
\hline C & 7.040138288 & 2.200912273 & -0.044208665 \\
\hline $\mathrm{C}$ & 6.425204458 & 2.613833897 & 1.134725299 \\
\hline $\mathrm{H}$ & 6.575258884 & 3.636395877 & 1.491283822 \\
\hline $\mathrm{C}$ & 5.577915075 & 1.759261719 & 1.866743146 \\
\hline $\mathrm{C}$ & 4.903836805 & 2.202768884 & 3.023699785 \\
\hline $\mathrm{H}$ & 5.031132185 & 3.238058868 & 3.349996508 \\
\hline $\mathrm{C}$ & 4.062960272 & 1.360856767 & 3.744242939 \\
\hline $\mathrm{C}$ & 3.339790730 & 1.809655627 & 4.899418690 \\
\hline $\mathrm{H}$ & 3.458193233 & 2.845997094 & 5.223984886 \\
\hline $\mathrm{C}$ & 2.504540343 & 0.967975151 & 5.568206064 \\
\hline $\mathrm{H}$ & 1.954070545 & 1.326372379 & 6.440449394 \\
\hline $\mathrm{C}$ & 2.346292693 & -0.385858309 & 5.147571464 \\
\hline $\mathrm{H}$ & 1.676864949 & -1.044834523 & 5.703008694 \\
\hline $\mathrm{C}$ & 3.029762619 & -0.859179693 & 4.068073173 \\
\hline $\mathrm{H}$ & 2.912954587 & -1.893677709 & 3.738320504 \\
\hline C & 3.900570690 & -0.004651227 & 3.316736695 \\
\hline $\mathrm{C}$ & 4.561167454 & -0.450448796 & 2.175150403 \\
\hline
\end{tabular}




\begin{tabular}{|c|c|c|c|}
\hline $\mathrm{H}$ & 4.418217261 & -1.480161977 & 1.842362181 \\
\hline $\mathrm{C}$ & 5.382998316 & 0.403861440 & 1.417697151 \\
\hline $\mathrm{C}$ & -4.580984219 & 6.395866582 & 1.877163038 \\
\hline $\mathrm{H}$ & -4.368677595 & 7.429265402 & 2.156751498 \\
\hline$C$ & -3.678702475 & 5.695644598 & 1.091945267 \\
\hline $\mathrm{H}$ & -2.751950469 & 6.162697548 & 0.756047514 \\
\hline $\mathrm{C}$ & -3.927893731 & 4.364569962 & 0.730654527 \\
\hline C & -2.962267385 & 3.618142680 & -0.016514111 \\
\hline $\mathrm{C}$ & -2.142357437 & 2.964161881 & -0.605899126 \\
\hline $\mathrm{C}$ & -1.221267909 & 2.149018076 & -1.334219095 \\
\hline $\mathrm{C}$ & -0.314405201 & 2.743525871 & -2.237016136 \\
\hline $\mathrm{C}$ & -0.269428598 & 4.156405635 & -2.457289107 \\
\hline $\mathrm{H}$ & -0.954779059 & 4.789841657 & -1.893686950 \\
\hline $\mathrm{C}$ & 0.617363807 & 4.702293598 & -3.339965622 \\
\hline $\mathrm{H}$ & 0.637531948 & 5.783517163 & -3.488322116 \\
\hline $\mathrm{C}$ & 1.533240375 & 3.875186117 & -4.054904965 \\
\hline $\mathrm{H}$ & 2.243612040 & 4.332175700 & -4.746531732 \\
\hline C & 1.510868336 & 2.525495644 & -3.886222402 \\
\hline $\mathrm{H}$ & 2.198130411 & 1.884842122 & -4.443297521 \\
\hline $\mathrm{C}$ & 0.583919006 & 1.904207649 & -2.986594826 \\
\hline $\mathrm{C}$ & 0.526931499 & 0.521159911 & -2.836713018 \\
\hline $\mathrm{H}$ & 1.196250025 & -0.108790502 & -3.427157024 \\
\hline C & -0.395326485 & -0.092093058 & -1.966424771 \\
\hline $\mathrm{C}$ & -0.495600433 & -1.495603775 & -1.867769643 \\
\hline $\mathrm{H}$ & 0.159343406 & -2.116229696 & -2.482636056 \\
\hline $\mathrm{C}$ & -1.409661294 & -2.101942826 & -1.012247048 \\
\hline $\mathrm{C}$ & -1.550511750 & -3.527563880 & -0.931075403 \\
\hline $\mathrm{H}$ & -0.902210472 & -4.148837914 & -1.551589790 \\
\hline $\mathrm{C}$ & -2.472539825 & -4.089916286 & -0.102238999 \\
\hline $\mathrm{H}$ & -2.565664386 & -5.176365514 & -0.049968648 \\
\hline $\mathrm{C}$ & -3.304828003 & -3.274088920 & 0.720296160 \\
\hline $\mathrm{H}$ & -4.027682067 & -3.746399249 & 1.387745474 \\
\hline $\mathrm{C}$ & -3.190081747 & -1.916745925 & 0.689730267 \\
\hline $\mathrm{H}$ & -3.824897123 & -1.290361626 & 1.319090693 \\
\hline $\mathrm{C}$ & -2.252337338 & -1.277681673 & -0.185062254 \\
\hline $\mathrm{C}$ & -2.160878023 & 0.108512583 & -0.276957380 \\
\hline $\mathrm{H}$ & -2.810616317 & 0.729052068 & 0.341311157 \\
\hline $\mathrm{C}$ & -1.267097045 & 0.731227008 & -1.167109360 \\
\hline $\mathrm{C}$ & -5.748936404 & 5.776457867 & 2.324938638 \\
\hline $\mathrm{H}$ & -6.447818054 & 6.329454350 & 2.953978760 \\
\hline $\mathrm{C}$ & -6.027433403 & 4.468783672 & 1.959674704 \\
\hline $\mathrm{H}$ & -6.951227911 & 3.989545259 & 2.286323813 \\
\hline $\mathrm{C}$ & -5.141274642 & 3.749531560 & 1.146171568 \\
\hline $\mathrm{C}$ & -5.463893553 & 2.426183563 & 0.707403455 \\
\hline C & -5.740274234 & 1.330601954 & 0.294326509 \\
\hline $\mathrm{C}$ & -5.999132541 & 0.014095070 & -0.198742828 \\
\hline
\end{tabular}




$\begin{array}{lrrr}\mathrm{C} & -6.816814585 & -0.867476282 & 0.539571818 \\ \mathrm{C} & -7.416785598 & -0.493888575 & 1.783452566 \\ \mathrm{H} & -7.234781308 & 0.510123721 & 2.169107978 \\ \mathrm{C} & -8.200941398 & -1.370152760 & 2.476830548 \\ \mathrm{H} & -8.647367537 & -1.063849999 & 3.424491542 \\ \mathrm{C} & -8.453419203 & -2.678454166 & 1.968227218 \\ \mathrm{H} & -9.092468344 & -3.357342533 & 2.535330128 \\ \mathrm{C} & -7.887655615 & -3.077883361 & 0.797335279 \\ \mathrm{H} & -8.062995499 & -4.083467189 & 0.407010734 \\ \mathrm{C} & -7.040138288 & -2.200912273 & 0.044208665 \\ \mathrm{C} & -6.425204458 & -2.613833897 & -1.134725299 \\ \mathrm{H} & -6.575258884 & -3.636395877 & -1.491283822 \\ \mathrm{C} & -5.577915075 & -1.759261719 & -1.866743146 \\ \mathrm{C} & -4.903836805 & -2.202768884 & -3.023699785 \\ \mathrm{H} & -5.031132185 & -3.238058868 & -3.349996508 \\ \mathrm{C} & -4.062960272 & -1.360856767 & -3.744242939 \\ \mathrm{C} & -3.339790730 & -1.809655627 & -4.899418690 \\ \mathrm{H} & -3.458193233 & -2.845997094 & -5.223984886 \\ \mathrm{C} & -2.504540343 & -0.967975151 & -5.568206064 \\ \mathrm{H} & -1.954070545 & -1.326372379 & -6.440449394 \\ \mathrm{C} & -2.346292693 & 0.385858309 & -5.147571464 \\ \mathrm{H} & -1.676864949 & 1.044834523 & -5.703008694 \\ \mathrm{C} & -3.029762619 & 0.859179693 & -4.068073173 \\ \mathrm{H} & -2.912954587 & 1.893677709 & -3.738320504 \\ \mathrm{C} & -3.900570690 & 0.004651227 & -3.316736695 \\ \mathrm{C} & -4.561167454 & 0.450448796 & -2.175150403 \\ \mathrm{H} & -4.418217261 & 1.480161977 & -1.842362181 \\ \mathrm{C} & -5.382998316 & -0.403861440 & -1.417697151\end{array}$

One-Ring shifted BET-B dimer
$\begin{array}{lrrr}\text { Nuclear Repulsion Energy } & =16100.1751128715 \\ \text { hartrees } \\ \text { C } & 5.376881531 & -5.330851791 & -3.428922802 \\ \text { H } & 5.334753832 & -6.283031959 & -3.959988313 \\ \text { C } & 4.414277847 & -5.033985680 & -2.476948655 \\ \text { H } & 3.609418588 & -5.739449370 & -2.267112643 \\ \text { C } & 4.446772192 & -3.816844568 & -1.782759490 \\ \text { C } & 3.411521248 & -3.478164648 & -0.854642959 \\ \text { C } & 2.526242894 & -3.159940247 & -0.104754053 \\ \text { C } & 1.521541942 & -2.742985673 & 0.822629471 \\ \text { C } & 0.789121303 & -3.705619710 & 1.549112576 \\ \text { C } & 1.006542242 & -5.110931854 & 1.392274385 \\ \text { H } & 1.762633756 & -5.439250814 & 0.677968394 \\ \text { C } & 0.284087966 & -6.018573024 & 2.111829521 \\ \text { H } & 0.461331821 & -7.087801804 & 1.979038604 \\ \text { C } & -0.718396502 & -5.585884757 & 3.029370089 \\ \text { H } & -1.296345963 & -6.323158130 & 3.589198204\end{array}$




\begin{tabular}{|c|c|c|c|}
\hline $\mathrm{C}$ & -0.943825386 & -4.257891205 & 3.219721430 \\
\hline $\mathrm{H}$ & -1.701322319 & -3.922037202 & 3.931840971 \\
\hline $\mathrm{C}$ & -0.196186059 & -3.266929095 & 2.503067301 \\
\hline $\mathrm{C}$ & -0.392339334 & -1.905661487 & 2.719879594 \\
\hline $\mathrm{H}$ & -1.129587347 & -1.585727998 & 3.460307986 \\
\hline $\mathrm{C}$ & 0.353862259 & -0.930680384 & 2.029491543 \\
\hline $\mathrm{C}$ & 0.199716025 & 0.444958386 & 2.300576301 \\
\hline $\mathrm{H}$ & -0.519347894 & 0.752110714 & 3.063609339 \\
\hline $\mathrm{C}$ & 0.939964134 & 1.408091234 & 1.622725974 \\
\hline $\mathrm{C}$ & 0.822849198 & 2.807772279 & 1.915983783 \\
\hline $\mathrm{H}$ & 0.108988941 & 3.117162512 & 2.682198018 \\
\hline $\mathrm{C}$ & 1.580259582 & 3.723830926 & 1.252273113 \\
\hline $\mathrm{H}$ & 1.483189114 & 4.786597148 & 1.483244031 \\
\hline $\mathrm{C}$ & 2.492175508 & 3.313807303 & 0.234858761 \\
\hline $\mathrm{H}$ & 3.080529870 & 4.062212696 & -0.298655316 \\
\hline $\mathrm{C}$ & 2.619901472 & 1.996671368 & -0.089671651 \\
\hline $\mathrm{H}$ & 3.321241683 & 1.682124370 & -0.865591807 \\
\hline C & 1.863291113 & 0.993176303 & 0.598491169 \\
\hline $\mathrm{C}$ & 2.022648706 & -0.362670918 & 0.325310812 \\
\hline $\mathrm{H}$ & 2.738599020 & -0.668247508 & -0.439509477 \\
\hline $\mathrm{C}$ & 1.307283045 & -1.346420396 & 1.032047803 \\
\hline $\mathrm{C}$ & 6.388753921 & -4.412296527 & -3.712917419 \\
\hline $\mathrm{H}$ & 7.138941715 & -4.641104805 & -4.471503105 \\
\hline $\mathrm{C}$ & 6.454396359 & -3.212942974 & -3.021374039 \\
\hline $\mathrm{H}$ & 7.254804690 & -2.499161776 & -3.219861171 \\
\hline $\mathrm{C}$ & 5.505985320 & -2.902246811 & -2.037314743 \\
\hline $\mathrm{C}$ & 5.616633568 & -1.698596467 & -1.271264382 \\
\hline $\mathrm{C}$ & 5.720352749 & -0.713103222 & -0.589088965 \\
\hline $\mathrm{C}$ & 5.772808977 & 0.458379997 & 0.228340411 \\
\hline $\mathrm{C}$ & 6.376993700 & 1.634924318 & -0.263172326 \\
\hline $\mathrm{C}$ & 6.957618053 & 1.709018350 & -1.568606254 \\
\hline $\mathrm{H}$ & 6.929386685 & 0.819764861 & -2.199772278 \\
\hline $\mathrm{C}$ & 7.532487672 & 2.862845257 & -2.017739484 \\
\hline $\mathrm{H}$ & 7.966795948 & 2.897278541 & -3.018180662 \\
\hline $\mathrm{C}$ & 7.581048568 & 4.021395270 & -1.187390965 \\
\hline $\mathrm{H}$ & 8.052865835 & 4.931940207 & -1.560964137 \\
\hline $\mathrm{C}$ & 7.025116295 & 3.996789202 & 0.053960678 \\
\hline $\mathrm{H}$ & 7.046824477 & 4.884828123 & 0.690302159 \\
\hline $\mathrm{C}$ & 6.391694939 & 2.815300273 & 0.561331254 \\
\hline $\mathrm{C}$ & 5.785558268 & 2.792331281 & 1.814568636 \\
\hline $\mathrm{H}$ & 5.777726353 & 3.702534902 & 2.419937185 \\
\hline $\mathrm{C}$ & 5.147157061 & 1.637526580 & 2.307430724 \\
\hline C & 4.476210103 & 1.638226425 & 3.548179881 \\
\hline $\mathrm{H}$ & 4.442405110 & 2.565186802 & 4.126146593 \\
\hline C & 3.841196236 & 0.499552349 & 4.033206204 \\
\hline $\mathrm{C}$ & 3.120975280 & 0.496628218 & 5.274213392 \\
\hline
\end{tabular}




\begin{tabular}{|c|c|c|c|}
\hline $\mathrm{H}$ & 3.078069870 & 1.423425523 & 5.850907068 \\
\hline $\mathrm{C}$ & 2.488345478 & -0.627576723 & 5.709286453 \\
\hline $\mathrm{H}$ & 1.938453434 & -0.613529740 & 6.652401586 \\
\hline $\mathrm{C}$ & 2.545654802 & -1.834314296 & 4.950774162 \\
\hline $\mathrm{H}$ & 2.037481516 & -2.727421604 & 5.319167746 \\
\hline $\mathrm{C}$ & 3.235716403 & -1.881330623 & 3.776855391 \\
\hline $\mathrm{H}$ & 3.277921826 & -2.804245276 & 3.194170735 \\
\hline C & 3.896076239 & -0.717278271 & 3.265055520 \\
\hline $\mathrm{C}$ & 4.555100678 & -0.726572693 & 2.038701891 \\
\hline $\mathrm{H}$ & 4.570903410 & -1.649121498 & 1.455242395 \\
\hline $\mathrm{C}$ & 5.167007333 & 0.429754499 & 1.521531664 \\
\hline $\mathrm{C}$ & -5.376881531 & 5.330851791 & 3.428922802 \\
\hline $\mathrm{H}$ & -5.334753832 & 6.283031959 & 3.959988313 \\
\hline $\mathrm{C}$ & -4.414277847 & 5.033985680 & 2.476948655 \\
\hline $\mathrm{H}$ & -3.609418588 & 5.739449370 & 2.267112643 \\
\hline $\mathrm{C}$ & -4.446772192 & 3.816844568 & 1.782759490 \\
\hline $\mathrm{C}$ & -3.411521248 & 3.478164648 & 0.854642959 \\
\hline C & -2.526242894 & 3.159940247 & 0.104754053 \\
\hline $\mathrm{C}$ & -1.521541942 & 2.742985673 & -0.822629471 \\
\hline $\mathrm{C}$ & -0.789121303 & 3.705619710 & -1.549112576 \\
\hline $\mathrm{C}$ & -1.006542242 & 5.110931854 & -1.392274385 \\
\hline $\mathrm{H}$ & -1.762633756 & 5.439250814 & -0.677968394 \\
\hline $\mathrm{C}$ & -0.284087966 & 6.018573024 & -2.111829521 \\
\hline $\mathrm{H}$ & -0.461331821 & 7.087801804 & -1.979038604 \\
\hline $\mathrm{C}$ & 0.718396502 & 5.585884757 & -3.029370089 \\
\hline $\mathrm{H}$ & 1.296345963 & 6.323158130 & -3.589198204 \\
\hline $\mathrm{C}$ & 0.943825386 & 4.257891205 & -3.219721430 \\
\hline $\mathrm{H}$ & 1.701322319 & 3.922037202 & -3.931840971 \\
\hline $\mathrm{C}$ & 0.196186059 & 3.266929095 & -2.503067301 \\
\hline $\mathrm{C}$ & 0.392339334 & 1.905661487 & -2.719879594 \\
\hline $\mathrm{H}$ & 1.129587347 & 1.585727998 & -3.460307986 \\
\hline $\mathrm{C}$ & -0.353862259 & 0.930680384 & -2.029491543 \\
\hline C & -0.199716025 & -0.444958386 & -2.300576301 \\
\hline $\mathrm{H}$ & 0.519347894 & -0.752110714 & -3.063609339 \\
\hline C & -0.939964134 & -1.408091234 & -1.622725974 \\
\hline $\mathrm{C}$ & -0.822849198 & -2.807772279 & -1.915983783 \\
\hline $\mathrm{H}$ & -0.108988941 & -3.117162512 & -2.682198018 \\
\hline $\mathrm{C}$ & -1.580259582 & -3.723830926 & -1.252273113 \\
\hline $\mathrm{H}$ & -1.483189114 & -4.786597148 & -1.483244031 \\
\hline $\mathrm{C}$ & -2.492175508 & -3.313807303 & -0.234858761 \\
\hline $\mathrm{H}$ & -3.080529870 & -4.062212696 & 0.298655316 \\
\hline C & -2.619901472 & -1.996671368 & 0.089671651 \\
\hline $\mathrm{H}$ & -3.321241683 & -1.682124370 & 0.865591807 \\
\hline $\mathrm{C}$ & -1.863291113 & -0.993176303 & -0.598491169 \\
\hline C & -2.022648706 & 0.362670918 & -0.325310812 \\
\hline $\mathrm{H}$ & -2.738599020 & 0.668247508 & 0.439509477 \\
\hline
\end{tabular}




$\begin{array}{lrrr}\mathrm{C} & -1.307283045 & 1.346420396 & -1.032047803 \\ \mathrm{C} & -6.388753921 & 4.412296527 & 3.712917419 \\ \mathrm{H} & -7.138941715 & 4.641104805 & 4.471503105 \\ \mathrm{C} & -6.454396359 & 3.212942974 & 3.021374039 \\ \mathrm{H} & -7.254804690 & 2.499161776 & 3.219861171 \\ \mathrm{C} & -5.505985320 & 2.902246811 & 2.037314743 \\ \mathrm{C} & -5.616633568 & 1.698596467 & 1.271264382 \\ \mathrm{C} & -5.720352749 & 0.713103222 & 0.589088965 \\ \mathrm{C} & -5.772808977 & -0.458379997 & -0.228340411 \\ \mathrm{C} & -6.376993700 & -1.634924318 & 0.263172326 \\ \mathrm{C} & -6.957618053 & -1.709018350 & 1.568606254 \\ \mathrm{H} & -6.929386685 & -0.819764861 & 2.199772278 \\ \mathrm{C} & -7.532487672 & -2.862845257 & 2.017739484 \\ \mathrm{H} & -7.966795948 & -2.897278541 & 3.018180662 \\ \mathrm{C} & -7.581048568 & -4.021395270 & 1.187390965 \\ \mathrm{H} & -8.052865835 & -4.931940207 & 1.560964137 \\ \mathrm{C} & -7.025116295 & -3.996789202 & -0.053960678 \\ \mathrm{H} & -7.046824477 & -4.884828123 & -0.690302159 \\ \mathrm{C} & -6.391694939 & -2.815300273 & -0.561331254 \\ \mathrm{C} & -5.785558268 & -2.792331281 & -1.814568636 \\ \mathrm{H} & -5.777726353 & -3.702534902 & -2.419937185 \\ \mathrm{C} & -5.147157061 & -1.637526580 & -2.307430724 \\ \mathrm{C} & -4.476210103 & -1.638226425 & -3.548179881 \\ \mathrm{H} & -4.442405110 & -2.565186802 & -4.126146593 \\ \mathrm{C} & -3.841196236 & -0.499552349 & -4.033206204 \\ \mathrm{C} & -3.120975280 & -0.496628218 & -5.274213392 \\ \mathrm{H} & -3.078069870 & -1.423425523 & -5.850907068 \\ \mathrm{C} & -2.488345478 & 0.627576723 & -5.709286453 \\ \mathrm{H} & -1.938453434 & 0.613529740 & -6.652401586 \\ \mathrm{C} & -2.545654802 & 1.834314296 & -4.950774162 \\ \mathrm{H} & -2.037481516 & 2.727421604 & -5.319167746 \\ \mathrm{C} & -3.235716403 & 1.881330623 & -3.776855391 \\ \mathrm{H} & -3.277921826 & 2.804245276 & -3.194170735 \\ \mathrm{C} & -3.896076239 & 0.717278271 & -3.265055520 \\ \mathrm{C} & -4.555100678 & 0.726572693 & -2.038701891 \\ \mathrm{H} & -4.570903410 & 1.649121498 & -1.455242395 \\ \mathrm{C} & -5.167007333 & -0.429754499 & -1.521531664\end{array}$

